CLINICAL STUDY

\title{
Insulin secretion and sensitivity in the prediction of type 1 diabetes in children with advanced $\beta$-cell autoimmunity
}

\author{
Heli T Siljander ${ }^{1,2,3,4}$, Robert Hermann ${ }^{5}$, Anne Hekkala ${ }^{6}$, Jyrki Lähde ${ }^{4}$, Laura Tanner ${ }^{7}$, Päivi Keskinen ${ }^{4}$, \\ Jorma Ilonen $^{5,8}$, Olli Simell ${ }^{7}$, Riitta Veijola ${ }^{6}$ and Mikael Knip ${ }^{1,2,3,4}$ \\ ${ }^{1}$ Children's Hospital, University of Helsinki and Helsinki University Central Hospital, PO Box 22, FI-O0014 Helsinki, Finland, ${ }^{2}$ Diabetes and Obesity \\ Research Program and ${ }^{3}$ Folkhälsan Research Centre, University of Helsinki, Helsinki, Finland, ${ }^{4}$ Department of Paediatrics, Tampere University Hospital, \\ Tampere, Finland, ${ }^{5}$ Immunogenetics Laboratory, University of Turku, Turku, Finland, ${ }^{6}$ Department of Paediatrics, Institute of Clinical Medicine, \\ University of Oulu, Oulu, Finland, ${ }^{7}$ Department of Paediatrics, University of Turku, Turku, Finland and ${ }^{8}$ Department of Clinical Microbiology, \\ University of Eastern Finland, Kuopio, Finland \\ (Correspondence should be addressed to M Knip at Children's Hospital, University of Helsinki and Helsinki University Central Hospital; \\ Email: mikael.knip@helsinki.fi)
}

\begin{abstract}
Objective: Reduced early insulin response has been shown to predict type 1 diabetes (T1D) in firstdegree relatives of diabetic patients, while its role, as well as that of insulin resistance, has remained poorly defined in young children representing the general population. The predictive values of these markers and their relation to other risk factors of T1D were assessed in children with advanced $\beta$-cell autoimmunity, i.e. persistent positivity for two or more autoantibodies.

Design and methods: Intravenous glucose tolerance tests (IVGTTs) were carried out in 218 children with HLA-DQB1-conferred disease susceptibility and advanced $\beta$-cell autoimmunity. Baseline, metabolic and growth data were compared between children progressing to diabetes and those remaining unaffected. Hazard ratios for the disease predictors and the progression rate of T1D were assessed.

Results: Children developing T1D were younger at seroconversion, progressed more rapidly to advanced $\beta$-cell autoimmunity and had lower first-phase insulin response (FPIR) and homeostasis model assessment index for insulin resistance (HOMA-IR) than those remaining non-diabetic. The levels of HOMA-IR/FPIR, islet cell antibodies, insulin autoantibodies (IAA) and islet antigen 2 antibodies (IA-2A) were higher in progressors. BMI SDS, FPIR, age at IVGTT and levels of IAA and IA-2A were predictive markers for T1D.

Conclusions: Young age, higher BMI SDS, reduced FPIR and higher levels of IAA and IA-2A predicted T1D in young children with HLA-DQB1-conferred disease susceptibility and advanced $\beta$-cell autoimmunity. Disease risk estimates were successfully stratified by the assessment of metabolic status and BMI. The role of insulin resistance as an accelerator of the disease process was minor.
\end{abstract}

European Journal of Endocrinology 169 479-485

\section{Introduction}

The prediabetic phase of type 1 diabetes (T1D) is characterised by destruction of the pancreatic $\beta$-cells leading to reduced insulin release and decreased glucose tolerance. Diabetes-associated autoantibodies can be used as predictive markers of $\mathrm{T} 1 \mathrm{D}$, and the risk assessment may be stratified by studying the insulin secretion and insulin sensitivity among prediabetic first-degree relatives $(1,2,3,4)$. The first-phase insulin response (FPIR) observed during an intravenous glucose tolerance test (IVGTT) provides information on the readily releasable insulin stored in the secretory granules located close to the $\beta$-cell membrane (5). A reduced FPIR represents the first marker of impaired insulin secretion, and it may appear at an early stage of prediabetes $(6,7)$. It has been suggested that insulin resistance can modify the progression rate of preclinical diabetes $(8,9)$, implicating that increasing obesity in the childhood population might contribute to the rise in the incidence of T1D that has been observed over the last decades (10). We assessed the role of FPIR, homeostasis model assessment index for insulin resistance (HOMA-IR) and insulin release-adjusted insulin resistance (ratio of HOMA-IR to FPIR, i.e. relative insulin resistance) as the predictors of T1D, and their interactions with other risk indicators in young children with signs of advanced $\beta$-cell autoimmunity, recruited from the general population on the basis of HLA-DQB1-conferred disease susceptibility. 


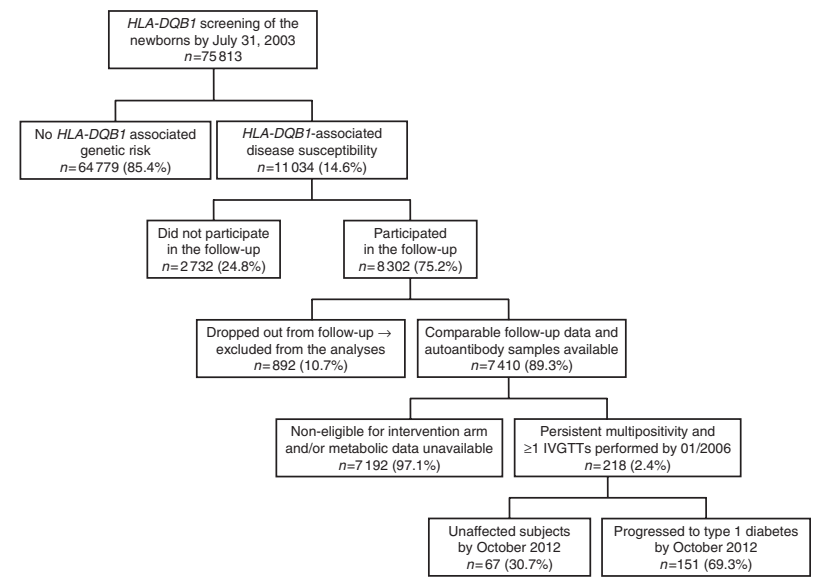

Figure 1 The DIPP study subcohort in the current analyses. IVGTT, intravenous glucose tolerance test.

\section{Subjects and methods}

\section{Subjects and study design}

The present study cohort comprises 218 participants in the Finnish Type 1 Diabetes Prediction and Prevention (DIPP) Study who were tested positive for more than one of four diabetes-associated autoantibodies (islet cell antibodies (ICA), autoantibodies against insulin (IAA),
GAD (GADA) and/or islet antigen 2 (IA-2A)) in at least two consecutive samples, taken with a minimum of 3 months apart and have undergone an IVGTT by January 1, 2006 (Fig. 1). Autoantibody analyses were performed as previously described (3). According to earlier analyses regarding the predictive value of autoantibodies and their combinations, the 5-year disease risk associated with multiple $(\geq 2)$ autoantibody positivity varied between 16.7 and $69.6 \%$ (3).

Participants were originally recruited to the DIPP Study as newborn infants, based on screening for risk-associated $H L A-D Q B 1$ genotypes defined by two risk-associated and two protective alleles. HLA-DR/DQ haplotypes relevant for risk determination were identified by using lanthanide-labelled sequence-specific oligonucleotides (11). As further analyses of DRB1, $D Q B 1$ and $D Q A 1$ loci revealed later that some of the children actually carried neutral or slightly protective HLA-DQB1 genotypes (Table 1), risk estimates for genotype-associated disease were subsequently categorised into five groups (12). INS (rs689) and PTPN22 (rs2476601) polymorphisms were genotyped by using the Sequenom (San Diego, CA, USA) platform (Genome Centre of Eastern Finland, University of Eastern Finland, Kuopio).

The majority $(179 ; 82.1 \%)$ of the children studied participated in a randomised, double-blinded

Table 1 Baseline data and variables related to growth, progression of autoimmunity and metabolic factors in children developing type 1 diabetes (T1D) and remaining unaffected.

\begin{tabular}{|c|c|c|c|c|}
\hline & All $(n=218)$ & Non-diabetic $(n=67)$ & T1D $(n=151)$ & $P$ value \\
\hline \multicolumn{5}{|l|}{ Baseline data $(n(\%))$} \\
\hline Gender, male & $129(59.2)$ & $39(58.2)$ & $90(59.6)$ & 0.847 \\
\hline Family history positive for T1D & $27(12.4)$ & $6(9.0)$ & $21(13.9)$ & 0.306 \\
\hline \multicolumn{4}{|l|}{$H L A-D Q B 1$-associated disease risk } & 0.698 \\
\hline Strongly increased & $75(34.4)$ & $19(28.4)$ & $56(37.1)$ & 0.453 \\
\hline Moderately increased & $120(55.0)$ & $40(59.7)$ & $80(53.0)$ & \\
\hline Neutral/slightly protective & $23(10.6)$ & $8(11.9)$ & $15(9.9)$ & \\
\hline $\begin{array}{l}\text { PTPN22 gene, rs2476601 CC } \\
\text { (vs CT/TT) }\end{array}$ & $74(34.1)$ & $17(25.4)$ & $57(38.0)$ & 0.070 \\
\hline INS gene, rs689 AA (vs AT/TT) & $170(78.7)$ & $51(76.1)$ & $119(79.9)$ & 0.534 \\
\hline \multicolumn{5}{|l|}{ Growth data (Median (range)) } \\
\hline Height-for-age (SDS) at IVGTT & $0.6(-3.1$ to 4.0$)$ & $0.6(-3.1$ to 2.4$)$ & $0.6(-1.2$ to 4.0$)$ & 0.551 \\
\hline BMI (SDS) at IVGTT & $0.3(-2.2$ to 5.5$)$ & $0.2(-1.6$ to 2.5$)$ & $0.4(-2.2$ to 5.5$)$ & 0.075 \\
\hline \multicolumn{5}{|l|}{ Delay (years) from: } \\
\hline Seroconversion to multipositivity & $0.0(0.0$ to 6.6$)$ & $0.3(0.0$ to 6.6$)$ & $0.0(0.0$ to 6.0$)$ & 0.003 \\
\hline $\begin{array}{l}\text { Multipositivity to persistent } \\
\text { multipositivity }\end{array}$ & $0.3(0.2$ to 3.3$)$ & $0.3(0.2$ to 3.3$)$ & $0.3(0.2$ to 3.3$)$ & 0.036 \\
\hline Persistent multipositivity to IVGTT & $0.2(0.0$ to 3.6$)$ & $0.3(0.0$ to 3.3$)$ & $0.2(0.0$ to 3.6$)$ & 0.020 \\
\hline Seroconversion to IVGTT & $0.9(0.2$ to 7.4$)$ & $1.2(0.4$ to 7.4$)$ & 0.7 (0.2 to 6.3$)$ & $<0.001$ \\
\hline \multicolumn{5}{|l|}{ Metabolic data } \\
\hline Fasting glucose $(\mathrm{mmol} / \mathrm{l})$ & 4.7 (2.6 to 6.3$)$ & 4.8 (3.7 to 6.0$)$ & 4.6 (2.6 to 6.3$)$ & 0.061 \\
\hline Fasting insulin (mU/l) & $3.8(0.1$ to 13.4$)$ & $4.1(1.1$ to 9.9$)$ & $3.8(0.1$ to 13.4$)$ & 0.084 \\
\hline Maximal glucose level (mmol/l) & $19.9(14.0$ to 27.6$)$ & $19.8(14.2$ to 26.3$)$ & 20.1 (14.0 to 27.6$)$ & 0.125 \\
\hline Maximal insulin level (mU/l) & 20.2 (3.1 to 157.2$)$ & 31.3 (6.5 to 157.2$)$ & 17.7 (3.1 to 115.6$)$ & $<0.001$ \\
\hline FPIR (mU/l) & 36.0 (5.0 to 273.6$)$ & 58.7 (12.1 to 273.6$)$ & 30.6 (5.0 to 213.6$)$ & $<0.001$ \\
\hline HOMA-IR $\left(\mathrm{mmol} \times \mathrm{mU} / \mathrm{I}^{2}\right)$ & 0.78 (0.02 to 3.27$)$ & $0.81(0.22$ to 2.64$)$ & 0.72 (0.02 to 3.27$)$ & 0.047 \\
\hline HOMA-IR/FPIR (mmol/l) & $0.021(0.003$ to 0.139$)$ & $0.015(0.003$ to 0.044$)$ & $0.024(0.003$ to 0.139$)$ & $<0.001$ \\
\hline
\end{tabular}

IVGTT, intravenous glucose tolerance test; PTPN22, protein tyrosine phosphatase non-receptor type 22; INS, insulin; FPIR, first-phase insulin response; HOMA-IR, homeostasis model assessment index for insulin resistance; HOMA-IR/FPIR, relative insulin resistance. 
intervention trial of administration of intranasal insulin vs placebo. The intervention proved to have no effect on the progression to T1D (13). Cases with T1D were diagnosed by the World Health Organization (WHO) criteria (14) and ascertained from the patient records of the University Hospitals of Turku, Oulu, and Tampere, and from the National Diabetes Register (15). Follow-up for T1D continued until the end of October 2012. Local ethics committees had approved the protocol of the DIPP study. Written informed consents were obtained from the guardians of the participants before the commencement of the study procedures.

\section{Metabolic and growth data}

The standardised Islet Cell Antibody Register Users Study (ICARUS) protocol was followed while performing the IVGTTs (1). Samples for plasma glucose and serum insulin assays were taken after overnight fasting. The fasting state (pre-infusion) samples were drawn 1 and 5 min before i.v. glucose infusion $(20 \%$ glucose solution given $0.5 \mathrm{~g}$ glucose $/ \mathrm{kg}$, maximal dose $35 \mathrm{~g}$; infusion time $3 \mathrm{~min} \pm 15 \mathrm{~s}$ ). Further samples were taken at 1, 3, 5 and $10 \mathrm{~min}$ after the infusion. Plasma glucose concentrations were measured by an enzymatic method (16) and serum intact insulin with an enzyme-linked two-site immunoassay (17). The intraassay coefficient of variation was $12.9 \%$ for the insulin analyses and $2.8 \%$ for the glucose assay.

The FPIR was defined as the sum of post-infusion insulin concentrations at 1 and $3 \mathrm{~min}$, while HOMA-IR represented the product of fasting glucose $(\mathrm{mmol} / \mathrm{l})$ and fasting insulin (mU/l) values divided by 22.5 (18). HOMA-IR:FPIR ratio (HOMA-IR:FPIR) represented the relative insulin resistance. Children's height and weight were measured at each follow-up visit. Values obtained (kilogram and centrimetre) were adjusted for age and gender $(19,20)$, and were given as SDS for height-for-age and BMI. Children with a BMI $>+2$ SDS were identified as overweight and those with a BMI $>+3$ SDS as obese (20).

\section{Statistical analyses}

Predictive Analytics Software (PASW Statistic 20.0 release, Armonk, NY, USA) was used for the statistical analyses. As the majority of the continuous variables had skewed distributions, mainly non-parametric tests, e.g. Mann-Whitney $U$ test, Kruskall-Wallis test, Wilcoxon signed-rank test and Spearman correlation analysis, were used. The $\chi^{2}$ test was applied for binary variables and the Kaplan-Meier log-rank test was used for the analyses of the rate of disease progression. Analyses of covariance were performed whenever appropriate. Hazard ratios (HR) for the factors potentially predictive for T1D were assessed in an ageadjusted manner with the Cox regression models. CIs were set at $95 \%$ and $P<0.05$ (two-tailed) was considered statistically significant. One child with unclear severe hyperinsulinemia was excluded from the analyses.

\section{Results}

Metabolic data were available for 218 subjects (129 males, 59.2\%), 151 (69.2\%) of whom developed T1D (progressors) at the median age of 6.4 years (range 1.6-16.8 years; Table 1). The median follow-up time since IVGTT for the 67 subjects remaining non-diabetic (non-progressors) was 8.8 years (range 6.8-15.0 years), and their age at the end of the follow-up was 13.3 years (8.5-17.0 years). Progressors and nonprogressors had similar distributions for baseline variables (Table 1), but progressors were younger at seroconversion and at all further time points regarding advanced seropositivity (Supplementary Table 1, see section on supplementary data given at the end of this article). Also, the delays from initial seroconversion to persistent multipositivity and IVGTT were shorter in progressors. As the age at IVGTT correlated significantly with metabolic markers (Spearman correlation coefficient, $r_{\mathrm{S}}=0.44, P<0.001$ for FPIR; $r_{\mathrm{S}}=0.41$, $P<0.001$ for HOMA-IR and $r_{\mathrm{S}}=-0.14, P=0.036$ for HOMA-IR/FPIR), age-adjustment was used in further analyses whenever applicable. Regarding autoantibodies, progressors had both higher numbers of positive autoantibodies and higher levels of ICA, IAA and IA-2A throughout the follow-up, except at the initial
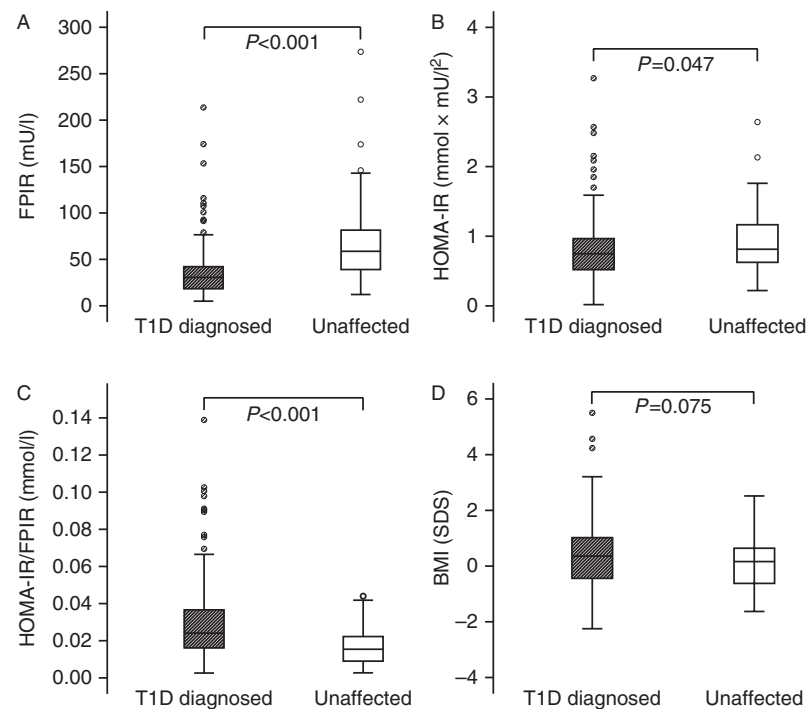

Figure 2 First-phase insulin response (FPIR; A), homeostasis model assessment index for insulin resistance (HOMA-IR; B), relative insulin resistance (HOMA-IR/FPIR; C) and BMI SDS (D) values (with $95 \% \mathrm{Cls}$ and medians) in children developing type 1 diabetes (T1D) and remaining unaffected. $P$ values for Mann-Whitney $U$ tests. 
seroconversion. Levels of GADA were comparable between the two groups during the whole follow-up (Supplementary Table 1).

In general, FPIR and HOMA-IR were higher in nonprogressors, while relative insulin resistance was higher in progressors (Table 1 and Fig. 2A, B, and C). Regarding HOMA-IR, the difference between the groups was small and the correlation between HOMA-IR and progression to T1D disappeared after adjustment for age $\left(r_{\mathrm{S}}=-0.04, P=0.57\right)$. As expected, HOMA-IR was correlated with BMI SDS $\left(r_{\mathrm{S}}=0.17, P=0.013\right)$. To study the predictive value of metabolic markers and BMI SDS, children were grouped by the quartiles of these variables, and the estimates of the disease progression rates of the different quartiles were compared with the log-rank test in the Kaplan-Meier analyses (Fig. 3A, B, C, and D). For FPIR, progression rate increased by the decreasing insulin secretion capacity, and all but the two middle quartiles differed significantly from each other. Regarding progression rates related to HOMA-IR, HOMA-IR/ FPIR and BMI SDS, mainly the highest quartiles differed from the other quartiles. The highest 5-year progression rates were observed for individuals belonging to

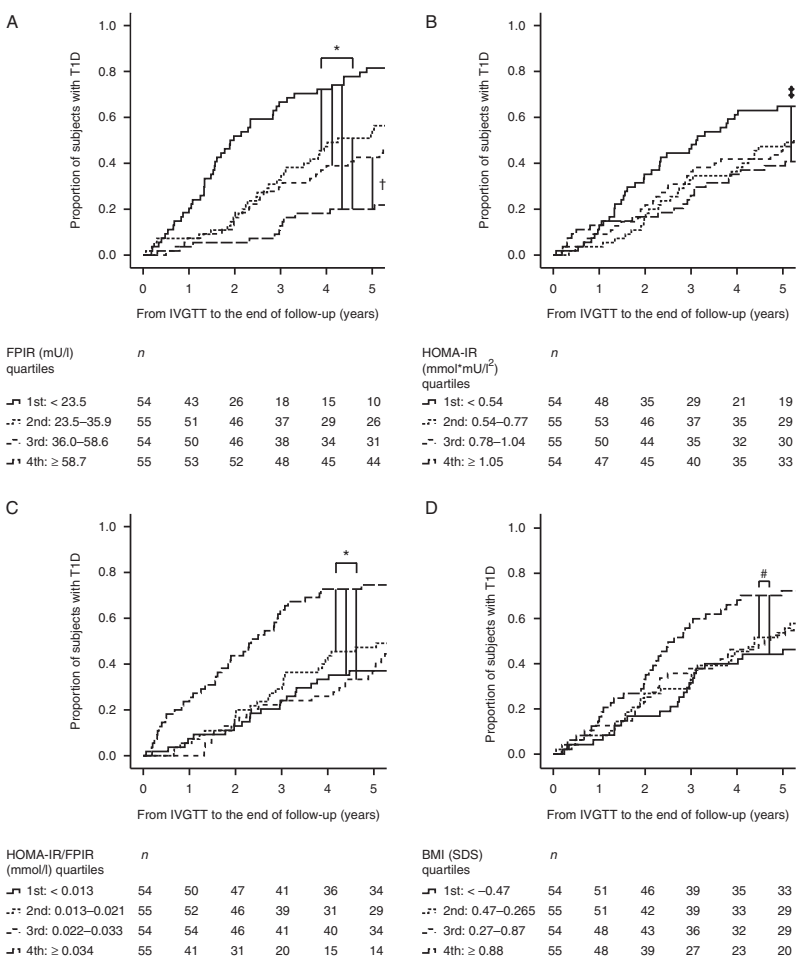

Figure 3 Progression to T1D according to the quartiles of firstphase insulin response (FPIR; A), homeostasis model assessment index for insulin resistance (HOMA-IR; $B$ ), relative insulin resistance (HOMA-IR/FPIR; C) and BMI SDS (D) in children developing type 1 diabetes (T1D) and remaining unaffected. The 1st quartile: continuous line; 2nd quartile: dotted line; 3rd quartile: sparse broken line; 4th quartile: dense broken line. IVGTT, intravenous glucose tolerance test. $P$ values for Kaplan-Meier log-rank tests. ${ }^{*} P<0.001$, ${ }^{\dagger} P=0.002,{ }^{\ddagger} P=0.01,{ }^{\sharp} P=0.025-0.047$. the lowest FPIR quartile (81.5\%; CI 71.1-91.9\%) and highest HOMA-IR/FPIR quartile (75.9\%; CI $64.5-87.3 \%$ ) and comparably, the lowest progression rates were recorded for those within the highest FPIR quartile (21.4\%; CI 10.6-32.2\%) and lowest HOMA-IR/ FPIR (37.0\%; CI 24.2-49.9\%) quartile.

As obesity had been hypothesised to have an accelerating effect on the prediabetic disease process, we assessed whether increased BMI could explain the progression to T1D. In the current study cohort, there were 203 children with their height and weight data recorded both at seroconversion and at IVGTT. At seroconversion, the median BMI for the 65 nonprogressors and 138 progressors were 0.2 SDS (range -1.7 to 2.2 SDS) and 0.5 SDS (range -1.8 to 5.5 SDS; $P=0.004)$ respectively. The median decrease in BMI during the follow-up from seroconversion to IVGTT was similar (0.2 SDS; $P=0.703)$ in both groups and did not correlate with progression to T1D $\left(r_{\mathrm{S}}=-0.034\right.$, $P=0.704)$. However, in the Kaplan-Meier analysis, the highest and the lowest BMI SDS quartile groups differed from each other only if the obese children $(n=6)$ were included in the analysis (Fig. 3D). Moreover, even if the BMI SDS was the main independent predictor for T1D in the Cox regression analysis (Table 2; HR 1.354, CI 1.137-1.613) in the whole cohort, this was not the case after excluding the obese children from the analysis. When the subcohort of the non-obese children $(n=212)$ was analysed, age at IVGTT, FPIR and IAA levels remained as significant predictors, while the ICA titre $(\mathrm{HR}=1.001$, CI 1.000-1.001; $P=0.006)$ replaced the IA-2A level as an independent predictor for T1D.

\section{Discussion}

We studied the prognostic value of metabolic data obtained soon after the confirmation of advanced $\beta$-cell autoimmunity in young children from the general population, carrying HLA-DQB1-conferred susceptibility to T1D. Our main aims were to test the hypothesis that obesity-related insulin resistance might modify the disease process, to confirm the role of a reduced FPIR as an important risk factor for rapid progression to T1D, and to assess the interactions and significance of the various disease risk factors present at the time of the IVGTT in children from the general population.

Although all children in the current study cohort had fulfilled the criteria of advanced $\beta$-cell autoimmunity before IVGTT, their progression rate to T1D was highly variable. In these children, the baseline factors, such as gender, HLA-DQB1 genotypes, insulin and PTPN22 gene polymorphisms, and the family history for T1D, could not identify individuals with rapid disease progression. This may be due to the inclusion criteria used, resulting in a relatively homogeneous study population, but may also reflect the idea that factors contributing to the initiation of the disease process might be different from those driving 
Table 2 Factors potentially predictive for type 1 diabetes (T1D) in the Cox univariate and multivariate analyses.

\begin{tabular}{|c|c|c|}
\hline Variables in the univariate analyses & Hazard ratio with $\mathrm{Cl}$ & $P$ value \\
\hline \multicolumn{3}{|l|}{ Background data } \\
\hline Gender male vs female* & $0.965(0.696-1.337)$ & 0.829 \\
\hline Family history for T1D positive vs negative* & $0.739(0.465-1.174)$ & 0.200 \\
\hline $\begin{array}{l}\text { HLA-DQB1 genotype by decreasing disease risk } \\
\text { class (see Table } 2)^{*}\end{array}$ & $0.876(0.671-1.143)$ & 0.330 \\
\hline INS genotype rs689 AA vs AT/TT* & $0.806(0.538-1.207)$ & 0.296 \\
\hline PTPN22 genotype rs2476601 CC vs CT/TT* & $0.719(0.516-1.001)$ & 0.051 \\
\hline Intervention study, participated vs refused & $0.928(0.608-1.416)$ & 0.728 \\
\hline \multicolumn{3}{|l|}{ Time-related variables } \\
\hline Age at seroconversion (years) & $0.837(0.748-0.936)$ & 0.001 \\
\hline Age at persistent multipositivity (years) & $0.795(0.716-0.884)$ & $<0.001$ \\
\hline Age at IVGTT (years)* & $0.778(0.701-0.862)$ & $<0.001$ \\
\hline Delay from seroconversion to IVGTT (years)* & $0.747(0.621-0.897)$ & 0.001 \\
\hline \multicolumn{3}{|l|}{ Data on autoantibodies } \\
\hline \multicolumn{3}{|l|}{ Autoantibody levels at IVGTT } \\
\hline ICA (JDFU)* & $1.000(1.000-1.001)$ & 0.001 \\
\hline $\mathrm{IAA}(\mathrm{RU})^{*}$ & $1.012(1.008-1.016)$ & $<0.001$ \\
\hline GADA $(R U)^{*}$ & $1.000(0.997-1.003)$ & 0.732 \\
\hline $\mathrm{IA}-2 \mathrm{~A}(\mathrm{RU})^{*}$ & $1.007(1.004-1.010)$ & $<0.001$ \\
\hline \multicolumn{3}{|l|}{ Number of positive autoantibodies } \\
\hline At seroconversion & $1.383(1.127-1.696)$ & 0.001 \\
\hline At seroconversion for multiple autoantibodies & $1.622(1.306-2.014)$ & $<0.001$ \\
\hline At persistent multipositivity & 1.669 (1.328-2.098) & $<0.001$ \\
\hline At IVGTT** & $1.643(1.343-2.011)$ & $<0.001$ \\
\hline \multicolumn{3}{|l|}{ Metabolic and growth related data } \\
\hline FPIR $(\mathrm{mU} / \mathrm{l})^{\star}$ & $0.978(0.970-0.985)$ & $<0.001$ \\
\hline HOMA-IR $\left(\mathrm{mmol} \times \mathrm{mU} / \mathrm{I}^{2}\right)^{*}$ & $0.719(0.484-1.069)$ & 0.103 \\
\hline HOMA-IR/FPIR $(\mu \mathrm{mol} / \mathrm{l})^{\star}$ & $1.019(1.013-1.026)$ & $<0.001$ \\
\hline Height-for-age (SDS) & $1.124(0.967-1.307)$ & 0.124 \\
\hline $\mathrm{BMI}(\mathrm{SDS})^{\star}$ & $1.280(1.092-1.501)$ & 0.002 \\
\hline \multicolumn{3}{|l|}{ Independent predictors of T1D (multivariate model) } \\
\hline BMI (SDS) & $1.354(1.137-1.613)$ & 0.001 \\
\hline Age at IVGTT (years) & $0.872(0.780-0.974)$ & 0.015 \\
\hline FPIR (mU/l) & $0.985(0.978-0.993)$ & $<0.001$ \\
\hline IAA at IVGTT (RU) & $1.010(1.005-1.014)$ & $<0.001$ \\
\hline IA-2A at IVGTT (RU) & $1.005(1.002-1.009)$ & 0.003 \\
\hline
\end{tabular}

the process towards overt disease. However, as previously shown $(2,3,6,21,22)$, several of the factors associated with the initiation of $\beta$-cell autoimmunity do contribute to the phenomena associated with the progression rate, such as age at initial seroconversion, the pace of progression towards persistent multipositivity and autoantibody levels. The current study confirmed the prior observations that progression to T1D is related to young age at seroconversion, early progression to multipositivity, higher numbers of detectable autoantibodies and to higher levels of ICA, IAA and IA-2A $(1,2,3)$.

Regarding IVGTT and metabolic data, the young and relatively variable age of the participants complicated the analyses. First of all, for HOMA-IR or HOMA-IR/FPIR scarcely any comparable age-adjusted data from a healthy, non-obese reference population was available, and second, as FPIR, HOMA-IR and HOMA-IR/FPIR correlated strongly with the age at IVGTT, age adjustments were required in the analyses. However, we confirmed that a low FPIR $(<24 \mathrm{mU} / \mathrm{l})$ identifies the future cases of T1D with high accuracy (5-year progression rate $>80 \%$ ), while children with reasonably high insulin secretion (FPIR $>59 \mathrm{mU} / \mathrm{l}$ ) remain unaffected for several years (5-year progression rate $21 \%$ ), although they already have signs of advanced $\beta$-cell autoimmunity.

The observation that HOMA-IR was remarkably low in the current cohort, and especially that it was slightly higher in non-progressors, implicates that the role of insulin resistance may be modest in the prediabetic process in young, mainly non-obese children. However, there were indications that both obesity-related insulin resistance and insulin resistance occurring in parallel with a reduced insulin secretion may modify the disease risk. These include the findings that HOMA-IR/FPIR was higher in progressors, it correlated positively with BMI SDS and the fact that in the Kaplan-Meier analyses assessing the rates of disease progression, the difference 
between the two highest BMI quartiles disappeared after excluding the obese children from the analysis, as did the significance of BMI as an independent predictor of T1D in the Cox regression analysis.

Our current findings are in line with the results of the British study on identical twins (23) and the data from the European Nicotinamide Diabetes Intervention Trial (ENDIT; (24)). In both studies, HOMA-IR was an independent predictor of T1D only in subjects with reduced FPIR, not in the whole study cohorts. The observation that both in the Diabetes Prevention TrialType 1 (DPT1) Study and in the Childhood Diabetes in Finland Study (DiMe) HOMA-IR and FPIR/HOMA-IR were predictive indicators for T1D $(2,4)$ might be explained by the older age of the study participants. In all, it seems that even if a low-grade insulin resistance is unable to shift glucose metabolism towards diabetes in individuals with normal FPIR, it might do so in individuals with reduced insulin release.

To conclude, the role of insulin resistance as an accelerator of the prediabetic disease process of T1D seems to be minor. However, the assessment of the metabolic status provides important information regarding the stratification of the disease risk in prediabetic children. This information can be applied in intervention trials aimed at preventing T1D: for the identification of the optimal trial participants, for the assessment of the metabolic changes during and after the treatment and as guidance in the interpretation of the study results.

\section{Supplementary data}

This is linked to the online version of the paper at http://dx.doi.org/ 10.1530/EJE-13-0206.

\section{Declaration of interest}

The authors declare that there is no conflict of interest that could be perceived as prejudicing the impartiality of the research reported.

\section{Funding}

This study was supported by the Special Public Grants for Medical Research at Tampere, Oulu, Turku, and Helsinki University Hospitals, the Academy of Finland (Centre of Excellence in Molecular Systems Immunology and Physiology Research 2012-17, Decision no. 250114), the Juvenile Diabetes Research Foundation International, the Novo-Nordisk Foundation, Finska Läkaresällskapet, the Foundation for Paediatric Research in Finland and the European Union Biomed.

\section{Author contribution statement}

H T Siljander analysed the data, wrote the first version of the manuscript and edited the manuscript. R Hermann was involved in the genetic analyses. H T Siljander, A Hekkala, J Lähde, L Tanner, P Keskinen and R Veijola were involved in the clinical follow-up of the study participants. J Ilonen, O Simell and M Knip are the Principal Investigators of the DIPP study. J Ilonen was responsible for the genetic analyses and M Knip and R Veijola for the autoantibody analyses. M Knip planned the study and contributed to the discussion. All authors reviewed and approved the manuscript. M Knip is the guarantor of this work and, as such, had full access to all the data in the study and takes responsibility for the integrity of the data and the accuracy of the data analysis.

\section{Acknowledgements}

The authors thank the staff members of the three clinical DIPP study centres and the personnel of the special DIPP laboratories, especially for their commitment to the study. Part of the data has been presented as abstracts at the annual meetings of International Society for Paediatric and Adolescent Diabetes in 2006 and 2008.

\section{References}

1 Bingley PJ. Interactions of age, islet cell antibodies, insulin autoantibodies, and first-phase insulin response in predicting risk of progression to IDDM in ICA + relatives: the ICARUS data set. Islet Cell Antibody Register Users Study. Diabetes 199645 1720-1728. (doi:10.2337/diabetes.45.12.1720)

2 Mrena S, Virtanen SM, Laippala P, Kulmala P, Hännilä ML, Åkerblom HK \& Knip M. Models for predicting type 1 diabetes in siblings of affected children. Diabetes Care 200629 662-667. (doi:10.2337/diacare.29.03.06.dc05-0774)

3 Siljander HT, Simell S, Hekkala A, Lähde J, Simell T, Vähäsalo P, Veijola R, Ilonen J, Simell O \& Knip M. Predictive characteristics of diabetes-associated autoantibodies among children with HLAconferred disease susceptibility in the general population. Diabetes 200958 2835-2842. (doi:10.2337/db08-1305)

$4 \mathrm{Xu} \mathrm{P}$, Wu Y, Zhu Y, Dagne G, Johnson G, Cuthbertson D, Krischer JP, Sosenko JM, Skyler JS \& Diabetes Prevention TrialType 1 (DPT-1) Study Group. Prognostic performance of metabolic indexes in predicting onset of type 1 diabetes. Diabetes Care 2010 33 2508-2513. (doi:10.2337/dc10-0802)

5 Daniel S, Noda M, Straub SG \& Sharp GWG. Identification of the docked granule pool responsible for the first phase of glucosestimulated insulin secretion. Diabetes 199948 1-5. (doi:10.2337/ diabetes.48.9.1686)

6 Veijola R, Vähäsalo P, Tuomilehto-Wolf E, Reijonen H, Kulmala P, Ilonen J, Åkerblom HK \& Knip M. Human leukocyte antigen identity and DQ risk alleles in autoantibody-positive siblings of children with IDDM are associated with reduced early insulin response. Diabetes 199544 1021-1028. (doi:10.2337/diabetes. 44.9.1021)

7 Keskinen P, Korhonen S, Kupila A, Veijola R, Erkkilä S, Savolainen H, Arvilommi P, Simell T, Ilonen J, Knip M et al. Firstphase insulin response in young healthy children at genetic and immunological risk for type I diabetes. Diabetologia 200245 1639-1648. (doi:10.1007/s00125-002-0981-8)

8 Fourlanos S, Narendran P, Byrnes GB, Colman PG \& Harrison LC. Insulin resistance is a risk factor for progression to type 1 diabetes. Diabetologia 200447 1661-1667. (doi:10.1007/s00125-004$1507-3)$

9 Xu P, Cuthbertson D, Greenbaum C, Palmer JP, Krischer JP \& Diabetes Prevention Trial-Type 1 Study Group. Role of insulin resistance in predicting progression to type 1 diabetes. Diabetes Care 200730 2314-2320. (doi:10.2337/dc06-2389)

10 Wilkin TJ. The accelerator hypothesis: weight gain as the missing link between type I and type II diabetes. Diabetologia $2001 \mathbf{4 4}$ 914-922. (doi:10.1007/s001250100548)

11 Ilonen J, Reijonen $\mathrm{H}$, Herva E, Sjöroos M, Iitiä A, Lövgren T, Veijola R, Knip M \& Åkerblom HK. Rapid HLA-DQB1 genotyping for four alleles in the assessment of risk for IDDM in the Finnish population. The Childhood Diabetes in Finland (DiMe) Study Group. Diabetes Care 199619 795-800. (doi:10.2337/diacare. 19.8.795)

12 Hekkala A, Ilonen J, Knip M, Veijola R \& Finnish Paediatric Diabetes Register Family history of diabetes and distribution of class II HLA genotypes in children with newly diagnosed type 1 diabetes: effect on diabetic ketoacidosis. European Journal of Endocrinology 2011 165 813-817. (doi:10.1530/EJE-11-0376) 
13 Näntö-Salonen K, Kupila A, Simell S, Siljander H, Salonsaari T, Hekkala A, Korhonen S, Erkkola R, Sipilä J, Haavisto L et al. Nasa insulin in prevention of type 1 diabetes in autoantibody-positive children with HLA-conferred disease susceptibility and recruited from the general population. Lancet $2008 \mathbf{3 7 2} 1746-1755$. (doi:10.1016/S0140-6736(08)61309-4)

14 World Health Organization. Definition, Diagnosis, and Classification of Diabetes Mellitus and its Complications: Report of a WHO Consultation. In World Health Organization publications, pp 66. Geneva, World Health Organization, 1999.

15 Parkkola A, Härkönen T, Ryhänen SJ, Ilonen J, Knip M \& Finnish Pediatric Diabetes Register. Extended family history of type 1 diabetes and phenotype and genotype of newly diagnosed children. Diabetes Care 201336 348-354. (doi:10.2337/dc12-0445)

16 Beach EF \& Turner JJ. An enzymatic method for glucose determination in body fluids. Clinical Chemistry $19584462-475$

17 Andersen L, Dinesen B, Jørgensen PN, Poulsen F \& Røder M. Enzyme immunoassay for intact human insulin in serum and plasma. Clinical Chemistry 199339 578-582.

18 Matthews DR, Hosker JP, Rudenski AS, Naylor BA, Treacher DF \& Turner RC. Homeostasis model assessment: insulin resistance and $\beta$-cell function from fasting plasma glucose and insulin concentrations in man. Diabetologia 198528 412-419. (doi:10.1007/ BF00280883)

19 WHO Multicentre Growth Reference Study Group. WHO Child Growth Standards: Length/height-for-age, weight-for-age, weightfor-length, weight-for-height and body mass index-for-age: Methods and development. In World Health Organization publications, pp 312. Web site: http://www.who.int/childgrowth/publications/en. Geneva: World Health Organization, 2006.
20 De Onis M, Onyango A, Borghi E, Siyam A, Nishida C \& Siekmann J. Development of a WHO growth reference for school-aged children and adolescents. Bulletin of the World Health Organization $2007 \mathbf{8 5}$ 661-668. (doi:10.2471/BLT.07.043497)

21 Hermann R, Lipponen K, Kiviniemi M, Kakko T, Veijola R, Simell O, Knip M \& Ilonen J. Lymphoid tyrosine phosphatase (LYP/PTPN22) Arg620Trp variant regulates insulin autoimmunity and progression to type 1 diabetes. Diabetologia $2006 \mathbf{4 9}$ 1198-1208. (doi:10.1007/s00125-006-0225-4)

22 Hermann R, Laine AP, Veijola R, Vahlberg T, Simell S, Lähde J, Simell O, Knip M \& Ilonen J. The effect of HLA class II, insulin and CTLA4 gene regions on the development of humoral $\beta$ cell autoimmunity. Diabetologia 200548 1766-1775. (doi:10.1007/ s00125-005-1844-x)

23 Hawa MI, Bonfanti R, Valeri C, Delli Castelli M, Beyan H \& Leslie RDG. No evidence for genetically determined alteration in insulin secretion or sensitivity predisposing to type 1 diabetes: a study of identical twins. Diabetes Care $2005 \mathbf{2 8} 1415-1418$. (doi:10.2337/diacare.28.6.1415)

24 Bingley PJ, Mahon JL, Gale EA \& the European Nicotinamide Diabetes Intervention Trial (ENDIT) Group. Insulin resistance and progression to type 1 diabetes in the European Nicotinamide Diabetes Intervention Trial (ENDIT). Diabetes Care $2008 \mathbf{3 1}$ 146-150. (doi:10.2337/dc07-0103)

Received 11 March 2013

Revised version received 2 July 2013

Accepted 29 July 2013 